\title{
Micro-environmental cross-talk in an organotypic human melanoma-in-skin model directs M2-like monocyte differentiation via IL-10
}

\author{
Elisabetta Michielon ${ }^{1}$ Marta López González ${ }^{2} \cdot J^{\prime}$ udith L. A. Burm ${ }^{1} \cdot$ Taco Waaijman $^{1} \cdot$ Ekaterina S. Jordanova $^{3}$ (1) . \\ Tanja D. de Gruij| ${ }^{2}$ (1) Susan Gibbs ${ }^{1,4}$ (1)
}

Received: 13 December 2019 / Accepted: 28 May 2020 / Published online: 7 June 2020

(c) The Author(s) 2020

\begin{abstract}
Preclinical assessment of novel therapies to fight cancer requires models that reflect the human physiology and immune response. Here, we established an in vitro three-dimensional (3D) reconstructed organotypic human melanoma-in-skin (Mel-RhS) model to investigate cellular and molecular features of tumor formation over a period of 6 weeks. Tumor nests developed over time at the epidermal-dermal junction and spread towards the dermis, in places disrupting the basement membrane. This coincided with secretion of matrix metalloproteinase 9 (MMP-9) by melanoma cells. These features resemble the initial stages of invasive melanoma. Interestingly, while the SK-MEL-28 cell line did not secrete detectable levels of interleukin-10 (IL-10) in traditional two-dimensional monolayers, it did express IL-10 in the 3D Mel-RhS, as did the surrounding keratinocytes and fibroblasts. This cellular cross-talk-induced secretion of IL-10 in the Mel-RhS indicated the generation of an immune suppressive microenvironment. Culture supernatants from Mel-RhS interfered with monocyte-todendritic-cell differentiation, leading to the development of M2-like macrophages, which was in part prevented by antibodymediated IL-10 blockade. Indeed, high-dimensional single-cell analysis revealed a shift within the monocyte population away from a CD $163^{+} \mathrm{PD}-\mathrm{L1}^{+} \mathrm{M} 2$-like phenotype upon IL-10 blockade. Thus, the 3D configuration of the Mel-RhS model revealed a role for IL-10 in immune escape through misdirected myeloid differentiation, which would have been missed in classical monolayer cultures.
\end{abstract}

Keywords Tumor progression $\cdot$ Reconstructed human skin $\cdot$ Melanoma $\cdot$ Tumor microenvironment $\cdot \mathrm{IL}-10 \cdot \mathrm{M} 2$ macrophages

Précis statement An in vitro human 3D melanoma-in-skin model recapitulates features of early invasion, stromal cross-talk, and myeloid immune suppression via IL-10 secretion.

Electronic supplementary material The online version of this article (https://doi.org/10.1007/s00262-020-02626-4) contains supplementary material, which is available to authorized users.

Susan Gibbs

s.gibbs@amsterdamumc.nl

1 Department of Molecular Cell Biology and Immunology, Amsterdam UMC, Vrije Universiteit, Amsterdam, The Netherlands

2 Department of Medical Oncology, Amsterdam UMC, Vrije Universiteit, Cancer Center Amsterdam, Amsterdam Infection \& Immunity Institute, Amsterdam, The Netherlands

\section{Introduction}

Melanoma is a deadly form of skin cancer which is caused by the malignant transformation of melanocytes. In its early phase, tumor cells are confined to the epidermis and melanomas can be successfully removed through surgical excision of the primary tumor lesion. However, tumor cells can undergo alterations that offer proliferative and survival

3 Center for Gynecologic Oncology Amsterdam (CGOA), Amsterdam UMC, Vrije Universiteit, Amsterdam, The Netherlands

4 Department of Oral Cell Biology, Academic Centre for Dentistry Amsterdam (ACTA), University of Amsterdam and Vrije Universiteit, Amsterdam, The Netherlands 
advantages and induce a switch towards an invasive phenotype. Once melanoma has spread, it becomes very difficult to treat and most patients eventually develop resistance to currently available treatments, including immunotherapy [1]. There was a major breakthrough in the field with the introduction of ipilimumab and nivolumab in 2011 and 2014, respectively [2]. Their unprecedented anti-melanoma efficacy demonstrated the presence of naturally occurring tumor-reactive $\mathrm{T}$ cells in the melanoma microenvironment that could successfully be activated and attack the tumor cells upon the blockade of immune checkpoints on their cell surface. Despite their impressive clinical success against melanoma, these drugs still remain ineffective in approximately half of the treated patients. One possible explanation for this may be resistance mechanisms at play in the tumor microenvironment (TME), in which immune suppressive myeloid cells have been implicated [3, 4]. Myeloid cells display extreme phenotypic plasticity and the TME is able to misdirect their differentiation from immune stimulatory subsets, like dendritic cells (DCs) and M1 macrophages, to regulatory subsets, such as M2 macrophages and myeloidderived suppressor cells (MDSCs), which can contribute to immune escape and tumor progression $[5,6]$. Therefore, understanding the processes involved in this myeloid suppression is crucial to the development of new therapeutic agents that can help overcome resistance to immune checkpoint blockade. Previous studies have investigated the crosstalk between myeloid and tumor cells using in vitro models based on two-dimensional (2D) monolayers of tumor cell lines [7]. However, this simple model poorly represents in vivo cancer behavior, as monocultures lack tissue context and the TME and do not take into consideration the relevant role of the stromal cells and the cross-talk between tumor cells and non-transformed cells in the induction of immune suppression [8-10]. Importantly, even though tumor cells initially trigger the expression of suppressive cytokines (such as interleukin-10, IL-10), stromal cells are often the main producers of these suppressive factors [11, 12], which in turn will result in the immune suppressive microenvironment found in cancer patients.

Although murine models have been extensively used to understand the biological mechanisms underlying melanoma metastasis and evasion of the immune system [13], fundamental differences in skin biology complicate extrapolation to the human situation [14]. Most importantly, animal models poorly predict the human immune response, with the result that quite often potential new drugs fail in the clinical trial setting [15]. Therefore, also in line with the 3Rs guidelines (reduction, refinement, and replacement of experimental animals) of the EU Directive 2010/63/EU [16], new melanoma models reproducing the human physiology and immune responses are urgently needed. The ultimate goal is hence to recreate, as closely as possible, the features of the TME, tumor growth, and metastasis in a physiologically relevant human three-dimensional (3D) model.

Over the past 10 years, diverse 3D in vitro melanoma models have been developed, as they are a good compromise between the lack of a microenvironment found in adherent cell cultures and the complexity of in vivo studies. Our previously described full-thickness reconstructed human skin $(\mathrm{RhS})$, consisting of an epidermal compartment of keratinocytes and melanocytes and a fibroblast-populated dermal equivalent [17-19], offers an attractive model to study invasive cell growth and behavior. The aim of this study was to develop a melanoma reconstructed human skin (Mel-RhS) model to investigate melanoma progression and invasion. Here, we showcase the ability of this novel model to recapitulate early invasive features and a previously recognized role for IL-10 in myeloid suppression and immune escape of metastatic melanoma [20], that is not adequately represented by classical 2D melanoma monolayer cultures [21].

\section{Materials and methods}

\section{Tissue and blood collection}

Human foreskin was obtained from healthy donors ( $<6$ years) undergoing circumcision and peripheral blood was collected from healthy adult donors (Sanquin Blood Supply Services, Amsterdam, The Netherlands). Melanoma patient-derived tissue samples were acquired from the Pathology Biobank, part of the VU University Medical Center Biobank. Histopathology of in situ and invasive melanoma tissue samples were confirmed by an experienced pathologist.

\section{Cell isolation and culture}

\section{Keratinocytes, melanocytes, and fibroblasts}

Epidermal cells (keratinocytes and melanocytes) and dermal fibroblasts were isolated from foreskins and cultured as previously described [22, 23]. Donor-matched fibroblasts and epidermal cells from first and second passage (p1-p2) were used in the experiments. Epidermal cells were co-cultured in Dulbecco's Modified Eagle Medium (DMEM; Lonza, Verviers, Belgium)/Ham's F-12 (Gibco, Grand Island, USA) in a $3: 1$ ratio, $1 \%$ penicillin/streptomycin (P/S; Invitrogen, Paisley, UK), 1\% UltroserG (UG; BioSepra S.A., Cergy-SaintChristophe, France), $0.1 \mu \mathrm{M}$ insulin (Sigma-Aldrich, St. Louis, USA), $1 \mu \mathrm{M}$ hydrocortisone (Sigma-Aldrich), $1 \mu \mathrm{M}$ isoproterenol (Sigma-Aldrich), and freshly supplemented $2 \mathrm{ng} / \mathrm{ml}$ keratinocyte growth factor (KGF; Sigma-Aldrich) at $37{ }^{\circ} \mathrm{C}$ and $7.5 \% \mathrm{CO}_{2}$. Dermal fibroblasts were cultured in DMEM with $1 \% \mathrm{P} / \mathrm{S}$ and $1 \% \mathrm{UG}$ at $37^{\circ} \mathrm{C}$ and $5 \% \mathrm{CO}_{2}$. 


\section{Melanoma cells}

The malignant melanoma SK-MEL-28 cell line was purchased from CLS Cell Lines Service GmbH (Eppelheim, Germany) and cultured in DMEM supplemented with $1 \%$ $\mathrm{P} / \mathrm{S}$ and $2 \% \mathrm{UG}$ at $37^{\circ} \mathrm{C}$ and $5 \% \mathrm{CO}_{2}$.

\section{Monocytes}

Peripheral blood mononuclear cells (PBMCs) were isolated from blood using lymphoprep (Axis-Shield Diagnostics, Dundee, Scotland) and a gradient centrifugation (1114547; Fresenius Kabi Norge AS, Halden, Norway). CD14 ${ }^{+}$monocytes were selected using magnetic activated cell sorting (MACS) with CD14 magnetic beads (Miltenyi Biotec, Bergisch Gladbach, Germany) following the manufacturer's protocol. The purity of the sorting was assessed by flow cytometry on FACS Fortessa (BD Biosciences, Franklin Lakes, USA) and routinely found to exceed 95\%. Monocytes were cultured in RPMI-1640 with HEPES and L-glutamine (BioWhittaker, Lonza) supplemented with $10 \%$ heat inactivated fetal calf serum (FCS, HyClone; GE Healthcare, Chicago, USA), $50 \mu \mathrm{M} \beta$-mercaptoethanol (2ME; Gibco), $100 \mathrm{IU} / \mathrm{ml}$ sodium-penicillin (Gibco), $100 \mu \mathrm{g} / \mathrm{ml}$ streptomycin (Gibco), and $2 \mathrm{mM}$ L-glutamine (Gibco).

\section{Construction of reconstructed human skin with or without melanoma cells}

$\mathrm{RhS}$ was constructed essentially as previously described [17] in $24 \mathrm{~mm}$ transwell plates (pore size of $8 \mu \mathrm{m}$; Corning, New York, USA). Briefly, dermal equivalents were constructed by mixing rat-tail collagen with 1:1 fibrinogen (Diagnostica Stago S.A.S., Asnieres sur Seine, France), Hank's Balanced Salt Solution (HBSS; Gibco) (HBSS was diluted 8-fold), and dermal fibroblasts $\left(1.3 \times 10^{5}\right.$ cells/gel $)$. Each hydrogel contained $0.5 \mathrm{U} / \mathrm{ml}$ thrombin (Merck KGaA, Darmstadt, Germany) to allow for fibrin formation. Epidermal cells were seeded onto the dermal equivalents at a density of $5 \times 10^{5}$ cells/gel. Mel-RhS models were created by seeding $2.5 \times 10^{4}$ SK-MEL-28 cells onto the gel $2 \mathrm{~h}$ prior to epidermal cell seeding. RhS and Mel-RhS were cultured in submerged conditions for 3 days and subsequently cultured at the air-liquid interface for 2, 4, or 6 weeks. During air-exposure, medium consisted of DMEM/Ham's F-12 (3:1), 1\% P/S, $0.2 \%$ $\mathrm{UG}, 1 \mu \mathrm{M}$ isoproterenol, $0.5 \mu \mathrm{M}$ hydrocortisone, $0.1 \mu \mathrm{M}$ insulin, $2 \mathrm{ng} / \mathrm{ml} \mathrm{KGF}, 1 \mathrm{ng} / \mathrm{ml}$ epidermal growth factor (EGF; Sigma-Aldrich), $10 \mathrm{mM}$ L-serine (Sigma-Aldrich), $10 \mu \mathrm{M}$ L-carnitine (Sigma-Aldrich), $25 \mu \mathrm{M}$ palmitic acid (Sigma-Aldrich), $7 \mu \mathrm{M}$ arachidonic acid (Sigma-Aldrich), $0.4 \mathrm{mM}$ ascorbic acid (Sigma-Aldrich), $15 \mu \mathrm{M}$ linoleic acid (Sigma-Aldrich), and $1 \mu \mathrm{M}$ vitamin E (Sigma-Aldrich), and was changed twice a week. Before harvesting, cultures were incubated $24 \mathrm{~h}$ in the above-mentioned medium but in the absence of hydrocortisone. Culture supernatant was collected and stored at $-20{ }^{\circ} \mathrm{C}$. Tissue sections were prepared for histological analysis.

\section{(Immuno)histochemistry}

Paraffin-embedded 5- $\mu$ m-thick tissue sections were used for morphological (hematoxylin and eosin staining, H\&E) and immunohistochemical analysis of collagen type IV (clone CIV22, Mon3251; Monosan, Uden, The Netherlands), Ki-67 (clone MIB-1, M7240; Dako, Glostrup, Denmark), laminin V (polyclonal, NB300-144; Novus Biologicals, Centennial, USA), Melan-A (clone A103, M7196; Dako), matrix metalloproteinase-9 (MMP-9; clone EP1255Y, ab137867; Abcam, Cambridge, UK), and proliferating cell nuclear antigen (PCNA; clone PC10, M0879; Dako). Briefly, sections were immersed in $0.01 \mathrm{M}$ sodium citrate buffer $(\mathrm{pH}$ 6.0) for collagen type IV, Ki-67, laminin V, and PCNA or $10 \mathrm{mM}$ Tris/1 mM EDTA buffer (pH 9.0) for Melan-A and MMP-9 for $15 \mathrm{~min}$ at $100{ }^{\circ} \mathrm{C}$, followed by slowly cooling to room temperature. For laminin $\mathrm{V}$ only, an additional protease digestion step with $4 \mathrm{mg} / \mathrm{ml}$ pepsin (Dako) in $0.2 \mathrm{M}$ $\mathrm{HCl}$ was performed for $15 \mathrm{~min}$ at room temperature. Sections were then washed in PBS, incubated with primary antibody for $1 \mathrm{~h}$ at room temperature, followed by incubation with BrightVision plus Poly-HRP-Anti-Mouse/Rabbit IgG (Immunologic, VWR International B.V., Breda, the Netherlands) and 3-amino-9-ethylcarbazole (AEC, Sigma-Aldrich) substrate, and a counterstain with hematoxylin. Stained tissue sections were photographed using a Nikon Eclipse 80i microscope (Nikon, Tokyo, Japan) with NIS Elements 4.13 software (Nikon).

\section{Fluorescent RNA in situ hybridization}

Paraffin-embedded 5- $\mu$ m-thick tissue sections were processed for single-molecule fluorescent RNA in situ hybridization (RNAish) according to the RNAScope ${ }^{\circledR}$ Multiplex Fluorescent V2 Assay instructions (Advanced Cell Diagnostics, Hayward, CA). For IL-10, the RNAscope ${ }^{\circledR}$ ProbeHs-IL10 (602051; Advanced Cell Diagnostics) was used. Either Opal 570 (PerkinElmer, Waltham, USA) or Opal 650 (PerkinElmer) was used to visualize IL-10. Fluorescent RNAish was combined with Melan-A (clone A103, M7196; Dako), pan-cytokeratin (clone C11, sc-8018 AF488; Santa Cruz Biotechnology, Dallas, USA), and DAPI (D3571; Invitrogen) immunofluorescent staining. Either Alexa Fluor 647-conjugated anti-mouse $\mathrm{IgG}(\mathrm{H}+\mathrm{L})$ (Invitrogen) or Opal 570 (PerkinElmer) was used to visualize Melan-A. Images were acquired on TCS SP8 STED $3 \times$ confocal microscope (Leica Microsystems B.V., Wetzlar, Germany) using a $\times 63$ oil-immersion lens. 


\section{ELISA}

Protein secretion into culture supernatants after $24 \mathrm{~h}$ was assessed by enzyme-linked immunosorbent assay (ELISA) as previously described [24] or according to the manufacturer's instructions. Interleukin-8 (IL-8) and IL-10 were measured using human IL-8 ELISA kit (Sanquin, Amsterdam, The Netherlands) and human IL-10 ELISA kit (Diaclone SAS, Besançon, France), respectively. Transforming growth factor beta 1 (TGF- $\beta 1$ ), granulocyte-macrophage colonystimulating factor (GM-CSF), and macrophage colony-stimulating factor (M-CSF) were detected using DuoSet ELISA kits (R\&D Systems, Minneapolis, USA). CCL2, CCL22, CXCL10, and vascular endothelial growth factor (VEGF) were quantified using the respective sandwich ELISA kit (R\&D Systems).

\section{Monocyte exposure to (Mel-)RhS-derived culture supernatant}

Monocytes $\left(4 \times 10^{4}\right.$ cells $)$ were cultured for 6 days either in the presence or absence of $30 \%$ (Mel-)RhS culture supernatant in a flat bottom 96-well plate in RPMI-1640 medium (Lonza) supplemented with $1000 \mathrm{IU} / \mathrm{ml}$ recombinant human GM-CSF (Immunotech, Prague, Czech Republic) and $20 \mathrm{ng} /$ $\mathrm{ml}$ recombinant human IL-4 (Strathmann Biotec, Hamburg, Germany). The same culturing procedure was followed in the IL-10 blocking experiment, but Mel-RhS supernatants were pre-treated for $30 \mathrm{~min}$ with either neutralizing antiIL-10 (clone 23738.11; Abcam) or IgG1 isotype (ICN Biomedicals, Irvine, USA) as a control.

\section{Flow cytometry}

After 6 days in culture, supernatant-exposed or unexposed monocyte-derived dendritic cells (moDCs) were harvested for fluorescence-activated cell sorting (FACS) analysis. Cell staining was performed using BDCA3-FITC (Miltenyi Biotec), CD1a-PE (BD Pharmingen, Franklin Lakes, USA), CD14-PerCPCy5.5 (BD Pharmingen), CD16-BV650 (BD Biosciences), CD163-BV421 (BD Horizon, Franklin Lakes, USA), PD-L1-APC (eBioscience, San Diego, USA), and PD-L2-BV711 (BD Horizon). Briefly, cells were collected and incubated for $10 \mathrm{~min}$ at $4{ }^{\circ} \mathrm{C}$ in $50 \mu \mathrm{l}$ of $1 \times$ EDTA $(500 \mathrm{nM})$. Collected cells were subsequently used for phenotypic readout by flow cytometry on LSR Fortessa (BD Biosciences). Analyses were performed with Kaluza flow cytometry analysis software (Beckman Coulter, Brea, USA) or FCS Express 6 (DeNovo Software, Glendale, USA).

\section{Statistical analysis}

All data are presented as mean \pm standard error of the mean (SEM). Statistical analysis was performed by means of a paired $t$ test or Pearson correlation using GraphPad Prism 7 software (GraphPad Software Inc., La Jolla, USA). Differences were considered to be significant when $p<0.05$. Supernatant from either seven (for CCL2, CCL22, IL-8, and GM-CSF), nine (for CXCL10, IL-10, and VEGF), or ten (for $\mathrm{M}-\mathrm{CSF}$ and TGF- $\beta 1$ ) independent experiments performed in duplicate was used for ELISA. Two different PBMC donors were used to assess expression of activation- and suppression-associated markers on moDCs.

\section{Results}

\section{The melanoma reconstructed human skin recapitulates the early stages of melanoma invasion}

Histological and phenotypic features of RhS and Mel-RhS models were compared to native full-thickness skin and patient-derived in situ and invasive melanoma skin lesions (Fig. 1, Supp. Fig. 1). The bi-layered RhS model consisted of a stratified epidermal layer on a fibroblast-populated fibrincollagen hydrogel (Supp. Fig. 1a). The epidermal layer comprised a compact basal cell layer, stratum spinosum, stratum granulosum, and stratum corneum, in line with the epidermis of native skin (Supp. Fig. 1a). Staining for the melanocyte lineage-specific marker Melan-A revealed the presence of melanocytes at the epidermal basal layer of both RhS (Supp. Fig. 1f) and native skin, and of tumor nests in the patient samples (Fig. 1a).

In the first stages of melanoma, tumor cells have not penetrated below the epidermis (melanoma in situ, Fig. 1a). However, with the progression of the disease, malignant cells were able to invade the basal membrane (BM) and spread vertically into the dermis (invasive melanoma, Fig. 1a). In line with this local progression of melanoma, Melan-A staining confirmed the expansion of the SK-MEL-28 melanoma cells in the Mel-RhS cultures (Fig. 1a), eventually leading to the partial disappearance of the epidermis at week 6 of air-exposed culture (Fig. 1a). Notably, particularly at week 4, melanoma cell aggregates were observed expanding into the dermis (Fig. 1a).

Since tumor cell proliferation is a key feature of stepwise neoplastic progression, we next inspected mitotically active cells by means of PCNA and Ki-67 staining (Fig. 1b, c, Supp. Figs. 1d, e, 2), both of which are prognostic biomarkers in developing melanoma [25]. In the native in situ melanoma sample, melanoma cells still expressed both proliferative markers to a certain extent, whereas in the invasive melanoma sample, both melanoma cells in the epidermis and 
Fig. 1 The melanoma reconstructed human skin (Mel-RhS) model recapitulates the initial stages of invasive melanoma. Comparison of morphology and phenotype between human native skin, melanoma biopsies, and Mel-RhS cultured for 2, 4, and 6 weeks at the air-liquid interface. a Melan-A staining shows melanoma growth and nest formation in the melanoma biopsies and in Mel-RhS over time. b PCNA and c Ki-67 staining shows a decrease in mitotically active cells in the Mel-RhS over time. d Collagen type IV staining shows a heterogeneous and disorganized protein deposition in the Mel-RhS, with expression and sporadic interruptions around melanoma nests (indicated by black arrows). e MMP-9 staining highlights its expression in SK-MEL-28 within the Mel$\mathrm{RhS}$. Representative stainings (paraffin-embedded 5- $\mu$ m-thick tissue sections) of at least four independent experiments (each with an intra-experiment replicate) are shown. Scale bar $=100 \mu \mathrm{m}$

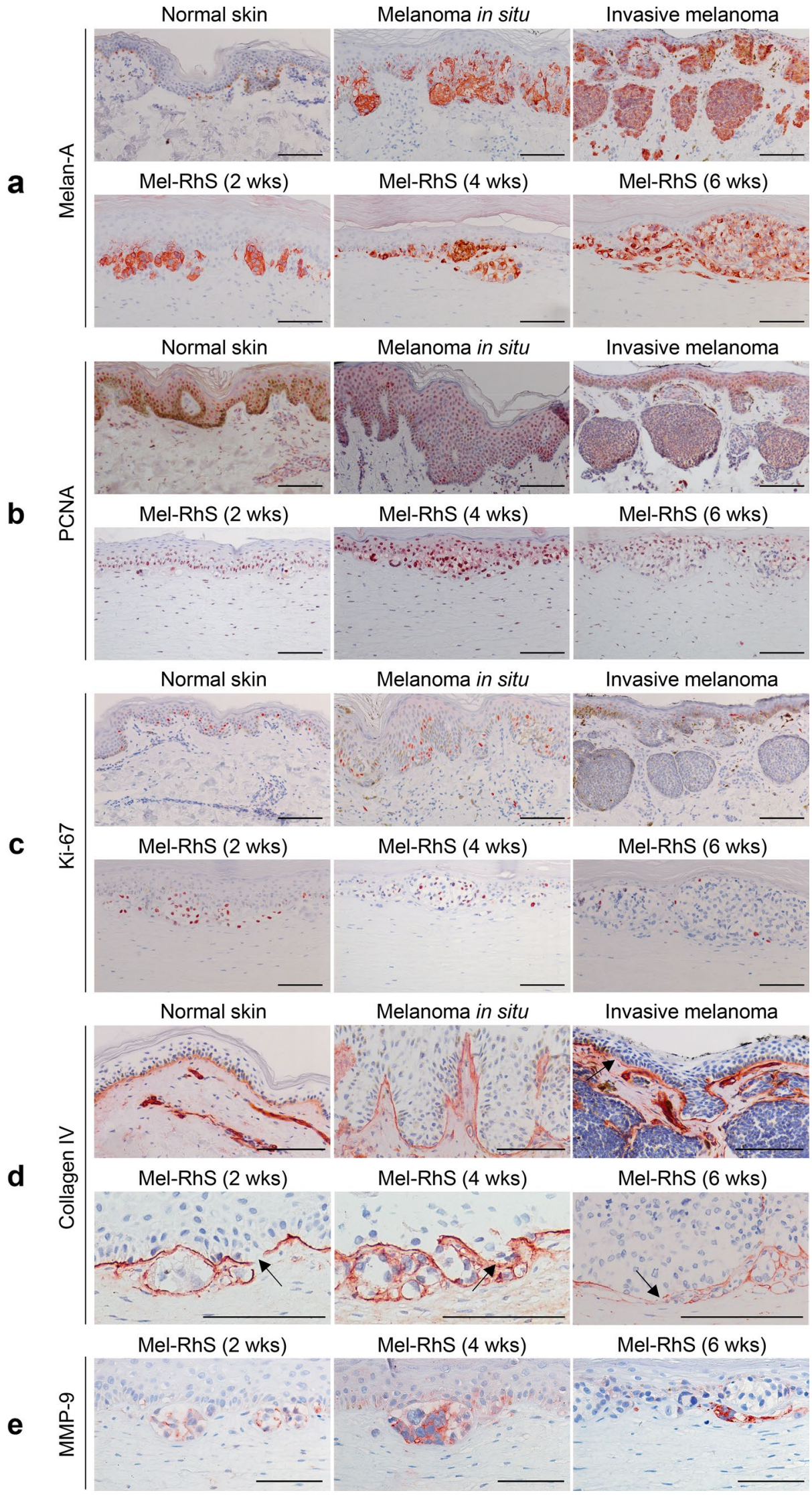


in the dermis showed very little mitotic activity (Fig. 1b, c). In the Mel-RhS model, at weeks 2 and 4, most of melanoma cells clearly expressed higher levels of both markers, whereas at week 6 , only a very limited number of melanoma cells were either PCNA or Ki-67 positive (Fig. 1b, c; Supp. Fig. 2).

\section{Melanoma cells disrupting the basal membrane coincides with secretion of MMP-9}

We next investigated expression of collagen type IV, a major component of the BM, which connects the epidermis to the dermis. In healthy human skin, a well-defined linear deposition of collagen type IV could be observed (Fig. 1d), indicating an intact BM. An intact BM was also observed in the RhS (Supp. Fig. 1c) and in the patient-derived in situ melanoma sample (Fig. 1d). In contrast, heterogeneous collagen type IV expression levels were detected at the dermal-epidermal junction and around the melanoma nests in the dermis of invasive melanoma (Fig. 1d). Similarly, in the Mel-RhS, the presence of melanoma cells led to the disruption of collagen type IV linear deposition and was accompanied by BM proteins surrounding the melanoma nests at different expression levels (Fig. 1d). At some sites, collagen type IV deposition appeared interrupted, as indicated by black arrows in Fig. 1d, which could indicate BM breakdown, a necessary step for melanoma invasion into the adjacent extracellular matrix. A similar heterogeneous expression pattern in the Mel-RhS was found for laminin V (Supp. Fig. 1b). The observation that BM perturbation was already observed after 2 weeks of culture could be due to the intrinsic invasion-related traits of the melanoma cell line used (SK-MEL-28), being BRAF/PTEN mutated and derived from a skin metastatic lesion. Therefore, although we did not investigate BM deposition at earlier time points, the used cell line apparently represents an already invasive state of melanoma development and will thus not fully recapitulate "normal" growth and development of melanoma in the skin model.

Next, the expression of MMP-9 was investigated. Clear expression of MMP-9 by the melanoma cells was observed at the melanoma/dermal border in the Mel-RhS (Fig. 1e), consistent with early invasive events and suggesting that the melanoma cells were actively degrading the BM through MMP-9 release. No differences in MMP-9 expression by fibroblasts and keratinocytes were observed between RhS and Mel-RhS (data not shown).

\section{Melanoma cells within the skin modulate the tissue microenvironment}

Tumors evade immunity via multiple mechanisms including the secretion of immunomodulatory cytokines. A statistically significant increase in the secretion of CXCL10, IL-10, M-CSF, TGF-B1, and VEGF $(* p=0.0225, * * * p<0.0001$, ${ }^{* *} p=0.0095, * * p=0.0058$, and $* p=0.0120$, respectively) in the Mel-RhS culture supernatant compared to the $\mathrm{RhS}$ control was found (Fig. 2). Notable de novo increases in CXCL10 and IL-10 secretion in Mel-RhS indicate a combined effector cell recruitment and immune suppressive capacity. Of note, no IL-10 was detected in the medium of SK-MEL-28 monocultures [21]. No difference was found in expression of CCL2, CCL22, IL-8, or GM-CSF between RhS and Mel-RhS (Fig. 2).
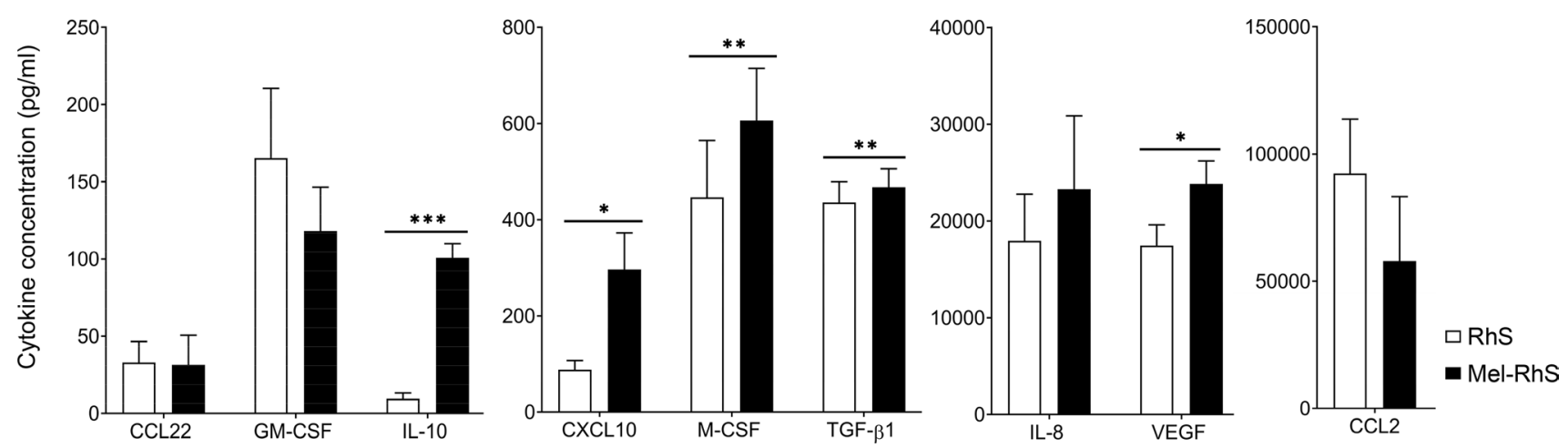

Fig. 2 Cytokine release by the melanoma reconstructed human skin (Mel-RhS) compared to its control (RhS). After 4 weeks culture at the air-liquid interface, medium was refreshed and culture supernatant was collected over a period of $24 \mathrm{~h}$. Cytokines were detected by means of ELISA. Results are shown as mean \pm SEM $\left({ }^{*} p<0.05\right.$,
$*_{*}^{*} p<0.01$, and $* * * p<0.001$; paired $t$ test; $N=7$ independent experiments performed in duplicate for CCL2, CCL22, IL-8, and GM-CSF; $N=9$ independent experiments performed in duplicate for CXCL10, IL-10, and VEGF; $N=10$ independent experiments performed in duplicate for M-CSF and TGF- $\beta 1$ ) 


\section{Melanoma model promotes an IL-10-dependent switch towards a M2-like phenotype during monocyte differentiation}

Given the increased IL-10 secretion in the medium of Mel$\mathrm{RhS}$ compared to $\mathrm{RhS}$, it was next investigated via fluorescent RNAish which cell types were responsible for its production (Fig. 3). Reflecting the low IL-10 secretion from the RhS skin model without the melanoma cells (assessed by ELISA), basal expression of IL-10 mRNA was found in keratinocytes and fibroblasts in the RhS (Fig. 3a). However, within the melanoma model, IL-10 mRNA was clearly increased compared to RhS (Fig. 3a) and detected in both epidermal keratinocytes, dermal fibroblasts, and melanoma nests (white arrows, Fig. 3b). Given the absence of IL-10 release from SK-MEL-28 cell line monocultures [21], this result suggests that the $3 \mathrm{D}$ skin microenvironment must have triggered IL-10 expression also in the melanoma cells, resulting in the up-regulation of IL-10 synthesis and subsequent release into the Mel-RhS culture supernatant.
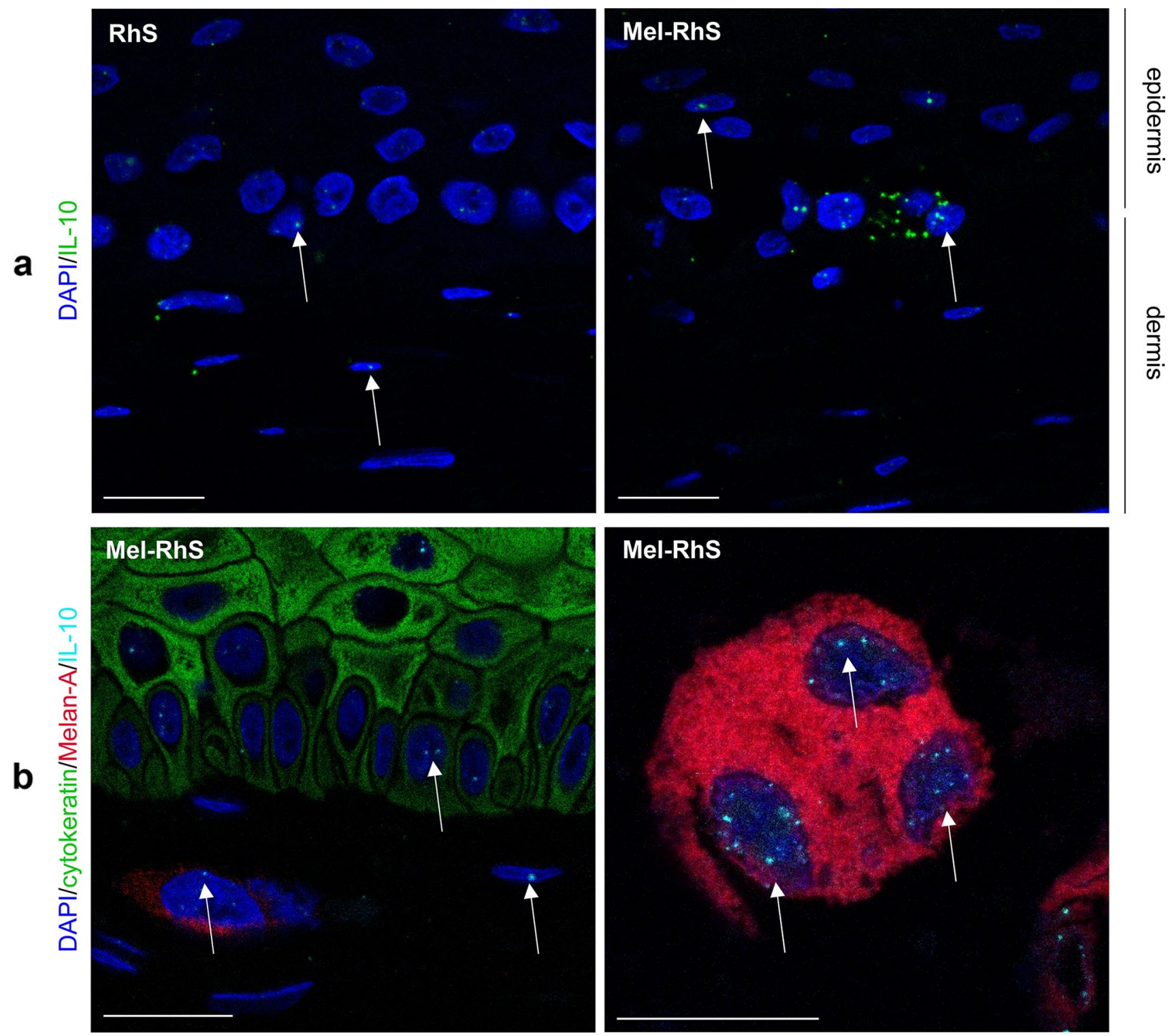

Fig. 3 Keratinocytes, fibroblasts, and melanoma cells produce IL-10 mRNA in the melanoma reconstructed human skin (MelRhS). a IL-10 mRNA was detected at single-cell level by fluorescent RNAish in the reconstructed human skin (RhS) and Mel-RhS model. Each dot (green) indicates one IL-10 mRNA molecule. b IL-10 fluorescent RNAish (cyan) was combined with Melan-A (red; melano-

cytes and melanoma cells), cytokeratin (green; keratinocytes), and DAPI (blue; nuclei of all cells, including fibroblasts) immunofluorescence staining. IL-10 mRNA spots in nuclei of keratinocytes (green) and melanoma cells (red) are clearly detectable, as well as in (typically elliptical) nuclei of unstained dermal fibroblasts, as indicated by white arrows. Scale bar $=20 \mu \mathrm{m}$ 

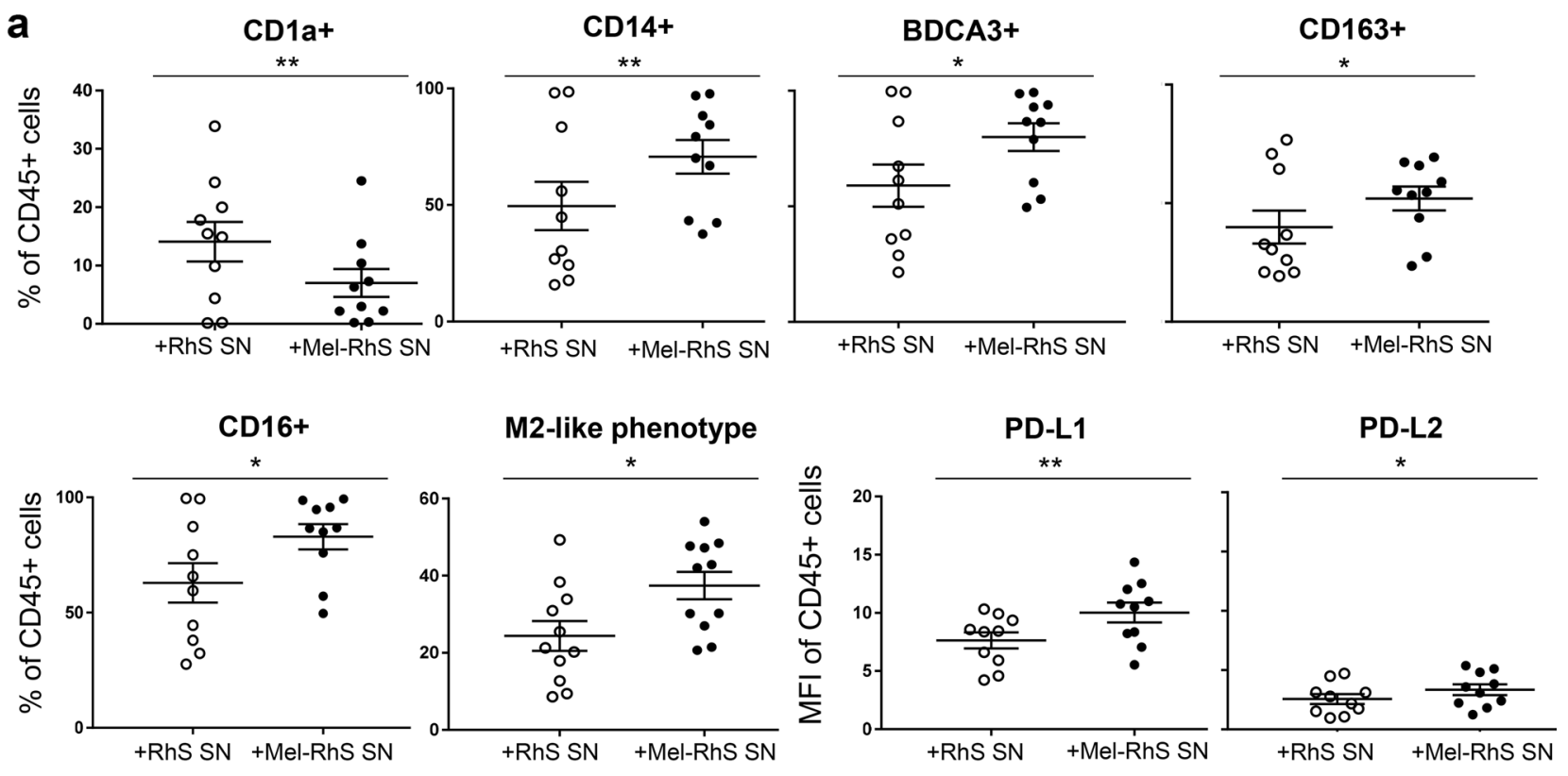

b
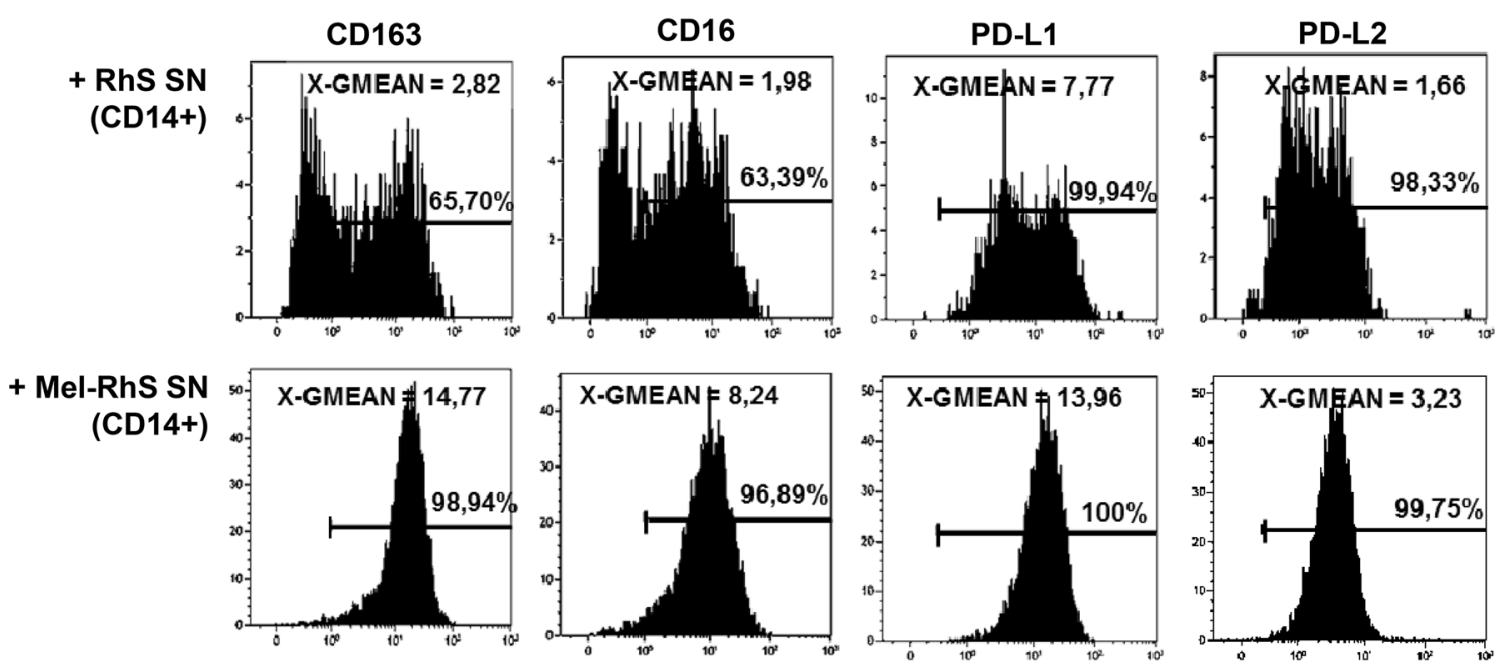

C
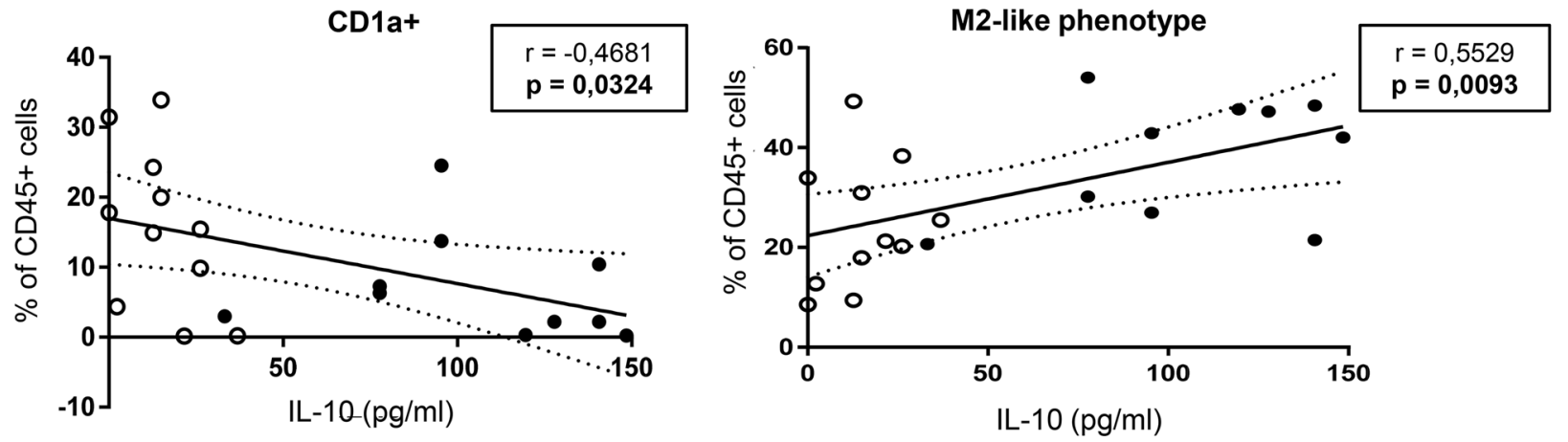

- RhS

- Mel-RhS 
4Fig. 4 moDC phenotype after exposure to melanoma reconstructed human skin (Mel-RhS) culture supernatants for 6 days compared to its control $(\mathrm{RhS})$ and its relation to IL-10 levels. a Frequency percentage of $\mathrm{CD}^{+} 5^{+}$cells expressing the surface markers CD1a, CD14, BDCA3, CD163, or CD16 and with an M2-like phenotype (defined as $\mathrm{CD} 14^{+} \mathrm{BDCA}^{+} \mathrm{CD} 163^{+} \mathrm{CD} 16^{+} \mathrm{PD}-\mathrm{L} 1^{+} \mathrm{PD}-\mathrm{L} 2^{+}$), and geometric mean intensity (MFI) of PD-L1 and PD-L2 in the $\mathrm{CD} 45^{+}$cells (i.e. monocytes cultured in the presence of the DC differentiation-inducing cytokines GM-CSF and IL-4), exposed to culture supernatants from either $\mathrm{RhS}(+\mathrm{RhS} \mathrm{SN}$; white circles) or Mel-RhS (+Mel-RhS $\mathrm{SN}$; black circles). Results are shown as mean \pm SEM $\left({ }^{*} p<0.05\right.$ and $* * p<0.01$; paired $t$ test; $N=10$ ). b Geometric mean intensity (MFI) and positive percentages of CD163, CD16, PD-L1, and PD-L2 expression on the $\mathrm{CD} 45^{+} \mathrm{CD} 14^{+}$cells upon exposure to either RhS- or Mel-RhS-derived supernatants (RhS SN and Mel-RhS SN, respectively). c Percentages of $\mathrm{CD}^{+}{ }^{+}$or M2-like cells (defined as $\mathrm{CD} 14^{+} \mathrm{BDCA}^{+} \mathrm{CD} 163^{+} \mathrm{CD} 16^{+} \mathrm{PD}-\mathrm{L} 1^{+} \mathrm{PD}-\mathrm{L} 2^{+}$) within the $\mathrm{CD} 45^{+}$ monocytic cell population and their correlation with the IL-10 levels found to be secreted in the supernatants derived from the $\mathrm{RhS}$ (white circles) or Mel-RhS (black circles) models. Results are shown with the $95 \%$ confidence bands of the best-fit line. Both $p$-value and Pearson $r$ value are shown

Due to the striking higher release of the M2 polarizing cytokine IL-10 by the melanoma model, it was next determined whether Mel-RhS-derived culture supernatants could interfere with the differentiation of monocytes into monocyte-derived dendritic cells (moDCs). We previously described a melanoma associated suppressed moDC phenotype, resembling an M2-like macrophage state with a lack of CD1 a expression and concerted up-regulation of the markers CD14, CD163, BDCA3/CD141, CD16, and PD-L1 [5]. Of note, these cells displayed a reduced capacity for the induction of type- 1 effector T cells $[5,7,21]$. When comparing the effects of the RhS versus the Mel-RhS on the differentiation into moDCs, our results clearly showed that monocytes cultured with the supernatants derived from Mel-RhS presented a similar immune suppressed phenotype, with significantly lower CD1a expression (** $p=0.0098)$ and higher levels of the M2-associated markers CD14, BDCA3, CD163, CD16, PD-L1, and PD-L2 $(* * p=0.0098, * p=0.0371, * p=0.0191$, $* p=0.0152, * * p=0.0083$, and $* p=0.0153$, respectively; Fig. 4a, b). Indeed, exposure to Mel-RhS supernatants resulted in an increase of the total percentage of cells showcasing a M2-like suppressed phenotype (* $p=0.0158)$, defined as $\mathrm{CD} 14^{+} \mathrm{BDCA}^{+} \mathrm{CD} 163^{+} \mathrm{CD} 16^{+} \mathrm{PD}-\mathrm{L} 1{ }^{+} \mathrm{PD}-\mathrm{L} 2^{+}$ (Fig. 4a). Furthermore, Fig. 4b shows the Mel-RhS supernatant-induced increase in expression levels of the surface markers CD163, CD16, PD-L1, and PD-L2 within the $\mathrm{CD} 14^{+}$population.

This suppressive effect of the Mel-RhS was directly correlated to IL-10 levels in the culture supernatant, resulting in decreased CD1a expression levels and increasing rates of cells with a M2-like phenotype at higher IL-10 concentrations (Fig. 4c).

Next, we assessed whether blocking IL-10 in Mel-RhS supernatants could prevent the skewing of monocytes to
M2-like macrophages. To this end, we performed a highdimensional t-Distributed Stochastic Neighbor Embedding ( $\mathrm{t}-\mathrm{SNE}$ ) analysis, based on the combined expression of the markers CD14, BDCA3, PD-L1, CD163, and CD16 (Fig. 5). Figure 5a shows a shift between two subsets within the conditioned monocyte population upon IL-10 neutralization. Gating on these subsets demonstrated that IL-10 blockade in the Mel-RhS supernatants led to a relative decrease of a sub-population with expression of CD14, BDCA3, PD-L1, CD163, and CD16 and an increase in a sub-population lacking these markers (Fig. 5b). Of note, CD16 expression followed a different expression pattern (Fig. 5b), whereas CD1a expression was not affected by the anti-IL-10 (data not shown). This indicates that other suppressive factors were most likely involved in the melanoma-induced changes in the expression of these two markers. Finally, IL-10 was shown to be at least in part responsible for the observed Mel-RhS-induced increase in M2-like cells (defined as $\mathrm{CD} 14^{+} \mathrm{BDCA}^{+}{ }^{+} \mathrm{CD} 163^{+} \mathrm{CD} 16^{+} \mathrm{PD}-\mathrm{L} 1^{+} \mathrm{PD}-\mathrm{L} 2{ }^{+}$), as IL-10 neutralizing antibodies led to a significant reduction in the frequencies of these cells in Mel-RhS supernatant-conditioned monocyte cultures $(* * p=0.0079$; Fig. 5c).

\section{Discussion}

Given the need for better in vitro testing platforms for antimelanoma therapeutic agents, we generated an in vitro full-thickness 3D organotypic Mel-RhS model displaying key features of early melanoma progression and invasion. Histopathologic features observed in patient-derived in situ and invasive melanoma tissue sections confirmed that the developed Mel-RhS model physiologically resembled the initial stages of invasive melanoma, in which melanoma aggregates start growing into the dermis. Importantly, the use of a single-cell suspension resulted in self-organized tumors growing into the dermis without the need to be seeded as pre-assembled spheroids. This was also accompanied by disruption of the BM, likely due to BM breakdown via MMP-9 produced by melanoma cells. MMP-9 secretion has been associated with tumor dissemination, as MMP-9 was expressed only by cell lines derived from advancedstage melanomas, whereas it was absent in cell lines derived from early-stage primary lesions [26]. MMP-9 expression by SK-MEL-28 cells is thus not surprising, given that the cell line was established from a metastatic site. Nevertheless, this would further confirm that the presented Mel-RhS model mimics early stages of invasive melanoma progression.

Consistent with previous studies reporting elevated levels of the immune suppressive factor IL-10 in the serum of advanced melanoma patients [27-29], we also showed its up-regulated secretion from the Mel-RhS. In vivo, IL-10 is involved in the formation and accumulation of 
a

$\lg G 1$

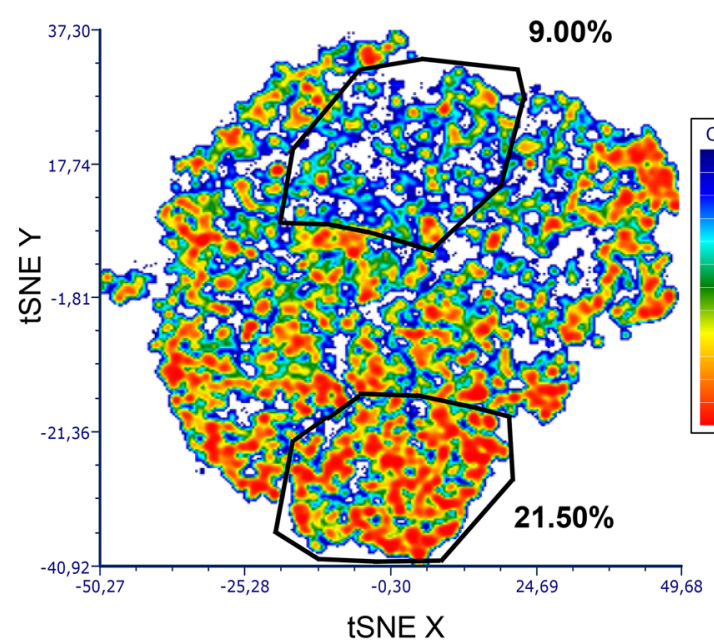

anti-IL-10

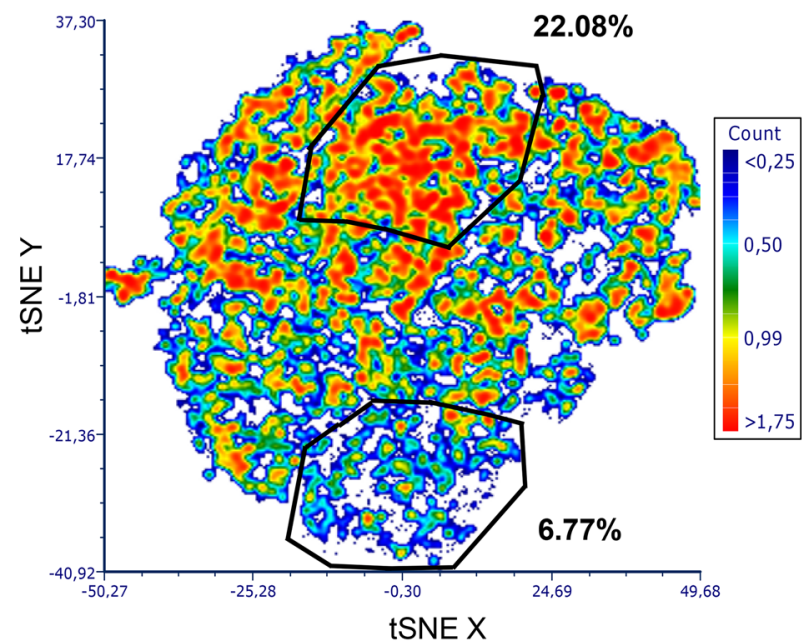

b
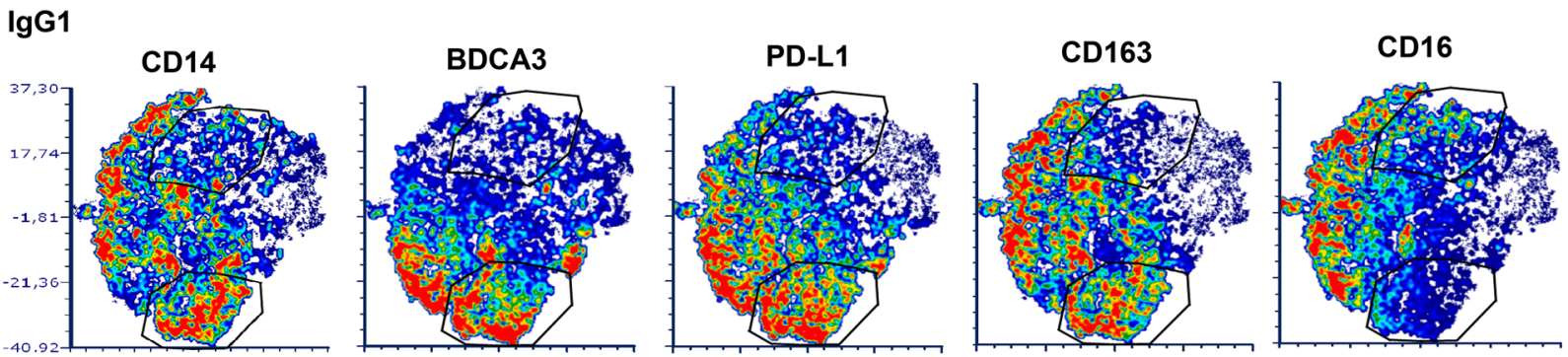

anti-IL-10
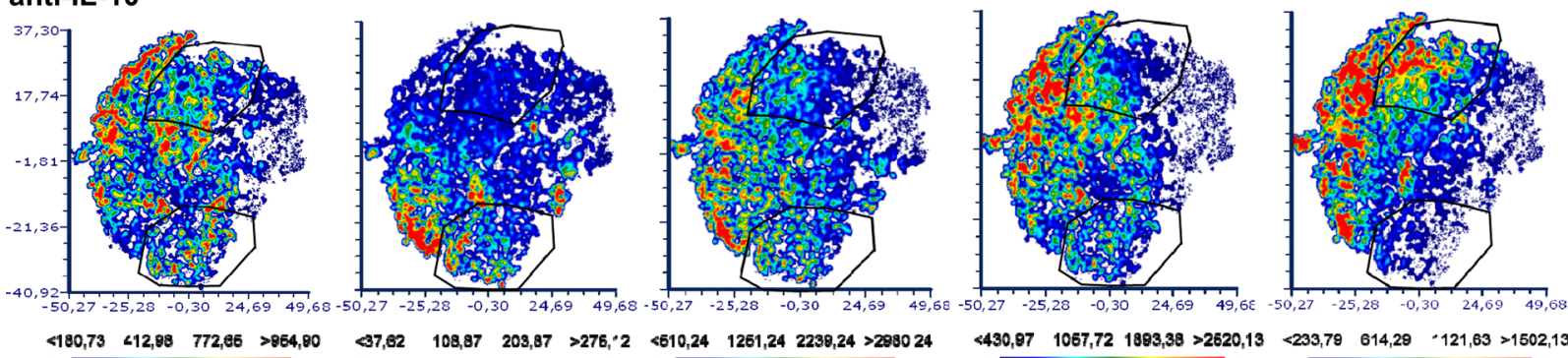

$<180,73 \quad 412,88 \quad 772,66 \quad>964,80$

$\begin{array}{lllllll}<7,62 & 108,87 & 203,87 & >276,-2 & <610,24 & 1261,24 & 2239,24\end{array}>298024$

$<430,97 \quad 1067,72 \quad 1893,38>2620,13$

$<233,79 \quad 614,29 \cdot 121,63>1502,13$

C M2-like phenotype

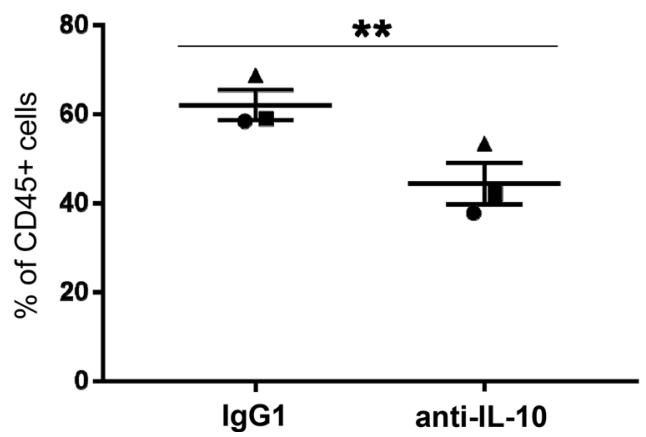


4Fig. 5 High-dimensional analysis of the phenotype of monocytes conditioned by supernatants derived from the melanoma reconstructed human skin (Mel-RhS) model cultured in the presence or absence of IL-10 neutralizing antibodies. a Differences in the t-SNE analyses between $\mathrm{IgG} 1$ and anti-IL-10 conditions. Two gates with shifting subsets between conditions are shown with the percentage of total $\mathrm{CD} 45^{+}$monocytes in that particular gate. b Differences between IgG1 and anti-IL-10 in the intensity and the distribution of expression of CD14, BDCA3, PD-L1, CD163, and CD16 in the t-SNE analysis. The same gates as in $\mathbf{a}$ are depicted in $\mathbf{b}$. $\mathbf{c}$ Percentage of M2-like cells (defined as $\mathrm{CD} 14^{+} \mathrm{BDCA} 3^{+} \mathrm{CD} 163^{+} \mathrm{CD} 16^{+} \mathrm{PD}-\mathrm{L} 1^{+} \mathrm{PD}-$ $\left.\mathrm{L}^{+}\right)$within the $\mathrm{CD} 45^{+}$cell population after incubation with MelRhS supernatant pre-treated with either IgG1 or anti-IL-10 $(N=3$; mean \pm SEM is shown)

tumor-associated macrophages (TAMs), which have features of alternatively activated M2 macrophages [30] and contribute to the suppression of anti-cancer T cell-mediated immune responses, in part through the expansion of regulatory T cells (Tregs) [5, 31-33]. Here, not only did we demonstrate that Mel-RhS supernatants were able to skew monocyte differentiation to a suppressive M2-like macrophage phenotype, but also that such an ability was indeed partly due to higher IL-10 release from the Mel-RhS cultures. The partial prevention of monocytes differentiating into M2-like macrophages upon IL-10 blockade in Mel-RhS supernatants suggests that other factors, such as M-CSF, TGF- $\beta 1$, and VEGF, whose secretion was also found to be up-regulated from the Mel-RhS, are likely to be also involved in this process. The described Mel-RhS model will be thus an important tool to uncover such factors and their role in skewing monocyte differentiation in the context of melanoma and possibly in additional immune suppression-related escape mechanisms.

Mel-RhS supernatant exposure of monocytes also led to a higher expression of suppression-associated markers (CD14, BCDA3, CD163, CD16, PD-L1, and PD-L2), as well as to a reduced CD1a expression, associated with migratory and monocyte-derived DC-like subsets. Interestingly, also in human melanoma lesions, CD1a expression on DCs was reported to be decreased in metastatic lesions as compared to primary lesions, which coincided with IL-10 expression specifically in metastatic lesions [20]. Consistent with this, supernatants from metastasisderived melanoma cells were significantly more effective in down-regulating CD1a on moDCs in an IL-10-dependent fashion in comparison to primary melanoma cultures. Interestingly, Gerlini et al. used supernatants from first passage metastatic melanomas to show this effect, which was lost in later passages. We also observed a lack of IL-10 release by established metastatic melanoma cell lines [21], whereas the incorporation of such a cell line (SK-MEL-28) into the Mel-RhS model resulted in marked up-regulation of IL-10 expression and release with melanoma cells, keratinocytes, and fibroblasts demonstrably expressing IL-10 mRNA in the Mel-RhS. This emphasizes that the presence of a physiologically relevant $3 \mathrm{D}$ configuration and skin microenvironment is able to rescue in vivo features of human melanomas that are otherwise lost in 2D cultures, suggesting, therefore, an interaction and thus cellular cross-talk between the different skin cell types. Taken together, these results suggest that the melanoma cells in the developed in vitro model were able to shape the skin microenvironment by promoting an immune suppressive secretome, comprising IL-10, the ability of which to skew in vitro monocyte differentiation towards a suppressive M2-like phenotype truly mimicked in vivo mechanisms of immune editing and evasion of metastatic melanoma [20].

Notably, although the observed suppressed M2-like monocytic phenotype also comprised CD16 expression, IL-10 blockade led to the loss of all other M2 markers, except for CD16, which was actually up-regulated and was co-expressed with lower levels of CD80 (not shown) and CD14. This phenotype is reminiscent of $\mathrm{CD} 14{ }^{\text {int }} \mathrm{CD} 16^{+}$ non-classical monocytes with pro-inflammatory functional characteristics [34]. This is of particular interest, since anti-CTLA4/ipilimumab was shown to engage non-classical monocytes ex vivo, resulting in antibody-dependent cell-mediated cytotoxicity (ADCC) of Tregs in melanoma patients [35]. Thus, IL-10 blockade in the metastatic melanoma environment may normalize DC development and allow for development and/or attraction of inflammatory non-classical monocytes, effectively facilitating immune checkpoint blockade by anti-PD1 and anti-CTLA4/ipilimumab, respectively $[4,35]$.

As with all in vitro models, our RhS-Mel does have its limitations and there is room for future developments. The model currently lacks integrated immune cells. An obvious next step will be to incorporate myeloid and $\mathrm{T}$ cells into the model to study melanoma-related immune suppressive effects in situ. Their incorporation will lead to the development of an in vitro tool most closely resembling the physiological TME for preclinical testing of immune modulatory therapeutics. However, our current 3D RhS-Mel model does allow for near-physiological cross-talk events between the keratinocyte/fibroblast/melanoma cell triad, thus enabling the study of the involvement of a myriad of secreted factors in the TME (e.g. IL-10) which are involved in melanoma-imposed immune suppression and which would otherwise have been missed in classic 2D melanoma cell line cultures. Thus, although clearly not yet optimized, even at this early stage of development, this 3D model allows for a more accurate and easy delineation of immune suppressive mechanisms at play in the melanoma TME. Furthermore, the addition of vasculature (with the aid of micro-fluidic organ-on-chip devices) will recapitulate more native tissue characteristics [36] and so allow the study of the interaction between melanoma cells, infiltrate, and the microvasculature. 
In this first study, we opted to focus on the well-established, characterized, and commercially available melanoma cell line, SK-MEL-28, to investigate the TME and the influence of the Mel-RhS secretome on monocyte differentiation. In follow-up studies, we will investigate other melanoma cell lines in this skin equivalent model to more extensively assess the relationship between TME and invasive potential.

In conclusion, our data showcase that by constructing a physiologically relevant 3D human melanoma-in-skin microenvironment, in vivo melanoma behavior and immune editing mechanisms may be uncovered, which might otherwise remain obscure.

Acknowledgements The authors would like to thank and acknowledge Professor Wolter J. Mooi for his assistance and discussions.

Author contribution EM, MLG, JLAB, TW, TDG, and SG contributed to the conception and design. EM, MLG, JLAB, TW, ESJ, TDG, and SG contributed to the development of methodologies. EM, MLG, and JLAB contributed to the acquisition of data. EM, MLG, JLA, TDG, and SG contributed to the analysis and interpretation of data. EM was responsible for writing the manuscript, while all authors contributed to manuscript editing and review, and approved the final manuscript version. SG and TDG were responsible for study supervision.

Funding This study was financed by the Dutch Government: Rijksdienst voor Ondernemend Nederland, EuroStars program, Project Number 2005392 .

\section{Compliance with ethical standards}

Conflict of interest The authors declare no potential conflicts of interest.

Ethical approval and ethical standards Tissue and blood samples were obtained under informed consent and used anonymously, in compliance with the VU University Medical Centre, Amsterdam UMC's ethical guidelines and the "Code for Proper Use of Human Tissues", as formulated by the Dutch Federation of Medical Scientific Organizations (https://www.federa.org).

Cell line authentication Upon receipt, the SK-MEL-28 cell line was expanded and stock vials were frozen. Cells resuscitated from the original vial were used up to 23 passages and were cultured for no longer than 6 months before thawing a new stock vial. The cell line was not re-authenticated but was tested and scored negative for Mycoplasma.

Open Access This article is licensed under a Creative Commons Attribution 4.0 International License, which permits use, sharing, adaptation, distribution and reproduction in any medium or format, as long as you give appropriate credit to the original author(s) and the source, provide a link to the Creative Commons licence, and indicate if changes were made. The images or other third party material in this article are included in the article's Creative Commons licence, unless indicated otherwise in a credit line to the material. If material is not included in the article's Creative Commons licence and your intended use is not permitted by statutory regulation or exceeds the permitted use, you will need to obtain permission directly from the copyright holder. To view a copy of this licence, visit http://creativecommons.org/licenses/by/4.0/.

\section{References}

1. Gorantla VC, Kirkwood JM (2014) State of melanoma: an historic overview of a field in transition. Hematol Oncol Clin North Am 28(3):415. https://doi.org/10.1016/j.hoc.2014.02.010

2. Ribas A, Wolchok JD (2018) Cancer immunotherapy using checkpoint blockade. Science 359:1350-1355. https://doi.org/10.1126/ science.aar4060

3. Tivnan A, Heilinger T, Lavelle EC, Prehn JHM (2017) Advances in immunotherapy for the treatment of glioblastoma. J Neurooncol 131(1):1-9. https://doi.org/10.1007/s11060-016-2299-2

4. Spranger S, Bao R, Gajewski TF (2015) Melanoma-intrinsic $\beta$-catenin signalling prevents anti-tumour immunity. Nature 523:231. https://doi.org/10.1038/nature14404

5. Lindenberg JJ, van de Ven R, Lougheed SM, Zomer A, Santegoets SJAM, Griffioen AW, Hooijberg E, van den Eertwegh AJM, Thijssen VL, Scheper RJ, Oosterhoff D, de Gruij1 TD (2013) Functional characterization of a STAT3-dependent dendritic cellderived $\mathrm{CD} 14^{+}$cell population arising upon IL-10-driven maturation. Oncoimmunology 2(4):e23837. https://doi.org/10.4161/ onci.23837

6. Tcyganov E, Mastio J, Chen E, Gabrilovich DI (2018) Plasticity of myeloid-derived suppressor cells in cancer. Curr Opin Immunol 51:76-82. https://doi.org/10.1016/j.coi.2018.03.009

7. Oosterhoff D, Lougheed S, van de Ven R, Lindenberg J, van Cruijsen H, Hiddingh L, Kroon J, van den Eertwegh AJM, Hangalapura B, Scheper RJ, de Gruijl TD (2012) Tumor-mediated inhibition of human dendritic cell differentiation and function is consistently counteracted by combined p38 MAPK and STAT3 inhibition. Oncoimmunology 1(5):649-658. https://doi. org/10.4161/onci.20365

8. Wang L, Yi T, Kortylewski M, Pardoll DM, Zeng D, Yu H (2009) IL-17 can promote tumor growth through an IL-6-Stat3 signaling pathway. J Exp Med 206:1457-1464. https://doi.org/10.1084/ jem.20090207

9. Wang L, Yi T, Zhang W, Pardoll DM, Yu H (2010) IL-17 enhances tumor development in carcinogen-induced skin cancer. Cancer Res 70(24):10112. https://doi.org/10.1158/0008-5472. CAN-10-0775

10. Binnewies M, Roberts EW, Kersten K, Chan V, Fearon DF, Merad M, Coussens LM, Gabrilovich DI, Ostrand-Rosenberg S, Hedrick CC, Vonderheide RH, Pittet MJ, Jain RK, Zou W, Howcroft TK, Woodhouse EC, Weinberg RA, Krummel MF (2018) Understanding the tumor immune microenvironment (TIME) for effective therapy. Nat Med 24(5):541-550. https://doi.org/10.1038/s4159 1-018-0014-x

11. Yu H, Kortylewski M, Pardoll D (2007) Crosstalk between cancer and immune cells: role of STAT3 in the tumour microenvironment. Nat Rev Immunol 7:41. https://doi.org/10.1038/nri1995

12. Yu H, Pardoll D, Jove R (2009) STATs in cancer inflammation and immunity: a leading role for STAT3. Nat Rev Cancer 9:798. https://doi.org/10.1038/nrc2734

13. Beaumont KA, Mohana-Kumaran N, Haass NK (2013) Modeling melanoma in vitro and in vivo. Healthcare 2(1):27-46. https://doi. org/10.3390/healthcare2010027

14. van den Broek LJ, Limandjaja GC, Niessen FB, Gibbs S (2014) Human hypertrophic and keloid scar models: principles, limitations and future challenges from a tissue engineering perspective. Exp Dermatol 23(6):382-386. https://doi.org/10.1111/exd.12419

15. Mak IW, Evaniew N, Ghert M (2014) Lost in translation: animal models and clinical trials in cancer treatment. Am J Transl Res 6(2):114-118

16. Directive 2010/63/EU of the European Parliament and of the Council of 22 September 2010 on the protection of animals used for scientific purposes 
17. Ouwehand K, Spiekstra SW, Waaijman T, Scheper RJ, de Gruijl TD, Gibbs S (2011) Technical Advance: langerhans cells derived from a human cell line in a full-thickness skin equivalent undergo allergen-induced maturation and migration. J Leukoc Biol 90(5):1027-1033. https://doi.org/10.1189/jlb.0610374

18. El Ghalbzouri A, Hensbergen P, Gibbs S, Kempenaar J, Rvd Schors, Ponec M (2003) Fibroblasts facilitate re-epithelialization in wounded human skin equivalents. Lab Invest 84(1):102-112. https://doi.org/10.1038/labinvest.3700014

19. Bil W, van der Bent SAS, Spiekstra SW, Nazmi K, Rustemeyer T, Gibbs S (2018) Comparison of the skin sensitization potential of 3 red and 2 black tattoo inks using interleukin-18 as a biomarker in a reconstructed human skin model. Contact Dermatitis 79(6):336-345. https://doi.org/10.1111/cod.13092

20. Gerlini G, Tun-Kyi A, Dudli C, Burg G, Pimpinelli N, Nestle FO (2004) Metastatic melanoma secreted IL-10 down-regulates CD1 molecules on dendritic cells in metastatic tumor lesions. Am J Pathol 165(6):1853-1863. https://doi.org/10.1016/S0002 $-9440(10) 63238-5$

21. López González M, Oosterhoff D, Lindenberg JJ, Milenova I, Lougheed SM, Martiáñez T, Dekker H, Quixabeira DCA, Hangalapura B, Joore J, Piersma SR, Cervera-Carrascon V, Santos JM, Scheper RJ, Verheul HMW, Jiménez CR, Van De Ven R, Hemminki A, Van Beusechem VW, De Gruijl TD (2019) Constitutively active GSK3 $\beta$ as a means to bolster dendritic cell functionality in the face of tumor-mediated immune suppression. Oncoimmunology. https://doi.org/10.1080/2162402x.2019.16311 19

22. Kosten IJ, Buskermolen JK, Spiekstra SW, de Gruijl TD, Gibbs S (2015) Gingiva equivalents secrete negligible amounts of key chemokines involved in langerhans cell migration compared to skin equivalents. Journal of immunology research 2015:11. https ://doi.org/10.1155/2015/627125

23. Waaijman T, Breetveld M, Ulrich M, Middelkoop E, Scheper RJ, Gibbs S (2010) Use of a collagen-elastin matrix as transport carrier system to transfer proliferating epidermal cells to human dermis in vitro. Cell Transplant 19(10):1339-1348. https://doi. org/10.3727/096368910X507196

24. Spiekstra SW, Toebak MJ, Sampat-Sardjoepersad S, Van Beek PJ, Boorsma DM, Stoof TJ, Von Blomberg BME, Scheper RJ, Bruynzeel DP, Rustemeyer T, Gibbs S (2005) Induction of cytokine (interleukin- $1 \alpha$ and tumor necrosis factor- $\alpha$ ) and chemokine (CCL20, CCL27, and CXCL8) alarm signals after allergen and irritant exposure. Exp Dermatol 14(2):109-116. https://doi.org/1 0.1111/j.0906-6705.2005.00226.x

25. de Wit NJ, van Muijen GN, Ruiter DJ (2004) Immunohistochemistry in melanocytic proliferative lesions. Histopathology 44(6):517-541. https://doi.org/10.1111/j.1365-2559.2004.01860.x

26. MacDougall JR, Matrisian LM (1995) Contributions of tumor and stromal matrix metalloproteinases to tumor progression, invasion and metastasis. Cancer Metastasis Rev 14(4):351-362. https://doi. org/10.1007/bf00690603

27. Boyano MD, Garcia-Vázquez MD, López-Michelena T, Gardeazabal J, Bilbao J, Cañavate ML, De Galdeano AG, Izu R,
Díaz-Ramón L, Raton JA, Díaz-Pérez JL (2000) Soluble interleukin-2 receptor, intercellular adhesion molecule-1 and interleukin-10 serum levels in patients with melanoma. Br J Cancer 83:847. https://doi.org/10.1054/bjoc.2000.1402

28. Dummer W, Bastian BC, Ernst N, Schänzle C, Schwaaf A, Bröcker E-B (1996) Interleukin-10 production in malignant melanoma: preferential detection of IL-10-secreting tumor cells in metastatic lesions. Int J Cancer 66(5):607-610. https ://doi.org/10.1002/(SICI)1097-0215(19960529)66:5\%3c607 :AID-IJC4\%3e3.0.CO;2-X

29. Dummer W, Becker JC, Schwaaf A, Leverkus M, Moll T, Bröcker EB (1995) Elevated serum levels of interleukin-10 in patients with metastatic malignant melanoma. Melanoma Res 5(1):67-68

30. Lewis CE, Harney AS, Pollard JW (2016) The Multifaceted Role of Perivascular Macrophages in Tumors. Cancer Cell 30(1):1825. https://doi.org/10.1016/j.ccell.2016.05.017

31. Mosser DM, Edwards JP (2008) Exploring the full spectrum of macrophage activation. Nat Rev Immunol 8:958. https://doi. org/10.1038/nri2448

32. Linde N, Gutschalk CM, Hoffmann C, Yilmaz D, Mueller MM (2012) Integrating macrophages into organotypic co-cultures: a $3 \mathrm{D}$ in vitro model to study tumor-associated macrophages. PLoS ONE 7(7):e40058. https://doi.org/10.1371/journal.pone.0040058

33. Lindenberg JJ, Oosterhoff D, Sombroek CC, Lougheed SM, Hooijberg E, Stam AGM, Santegoets SJAM, Tijssen HJ, Buter J, Pinedo HM, van den Eertwegh AJM, Scheper RJ, Koenen HJPM, van de Ven R, de Gruijl TD (2013) IL-10 conditioning of human skin affects the distribution of migratory dendritic cell subsets and functional T cell differentiation. PLoS ONE 8(7):e70237-e70237. https://doi.org/10.1371/journal.pone.0070237

34. Mukherjee R, Kanti Barman P, Kumar Thatoi P, Tripathy R, Kumar Das B, Ravindran B (2015) Non-classical monocytes display inflammatory features: validation in sepsis and systemic lupus erythematous. Sci Rep 5:13886. https://doi.org/10.1038/ srep13886

35. Romano E, Kusio-Kobialka M, Foukas PG, Baumgaertner $\mathrm{P}$, Meyer C, Ballabeni P, Michielin O, Weide B, Romero P, Speiser DE (2015) Ipilimumab-dependent cell-mediated cytotoxicity of regulatory $\mathrm{T}$ cells ex vivo by nonclassical monocytes in melanoma patients. Proc Natl Acad Sci 112:6140-6145. https://doi. org/10.1073/pnas.1417320112

36. Bourland J, Fradette J, Auger FA (2018) Tissue-engineered 3D melanoma model with blood and lymphatic capillaries for drug development. Sci Rep 8(1):13191. https://doi.org/10.1038/s4159 8-018-31502-6

Publisher's Note Springer Nature remains neutral with regard to jurisdictional claims in published maps and institutional affiliations. 